\title{
Wideband Analog Transmission System Based on the External Intensity Electro-Optic Modulator
}

\author{
Jiri Svarny
}

\begin{abstract}
The work deals with design and integration of an analog electro-optic transmission system suitable for some specialized tasks of diagnostics and measurements. The system is based on principle of external intensity modulation of fiber guided laser radiation. Besides wideband and almost lossless transmission the system tolerates extreme length of the transmitting medium and ensures ultimate galvanic barrier between the input and output.
\end{abstract}

Keywords-Electro-optic modulator, fiber optics, intensity modulation, transmission system, wideband transmission.

\section{INTRODUCTION}

In regard to a measuring device the transmission system forms an important and integral part of the measuring chain. Its task is to deliver a sensed signal from a sensor to an instrument with the highest possible fidelity. In most of applications a coaxial cable is quite satisfactory to fulfill the needs. However, the transmission system based on the coaxial cable suffers from certain limitations:

\section{A. Signal Attenuation}

As the frequency range is low and the cable is not too long the attenuation is negligible. The higher the frequency of the signal and the longer the cable is the more severe attenuation the metallic transmission system demonstrates [1]. In the $\mathrm{GHz}$ range the attenuation can easily reach several $\mathrm{dB} / \mathrm{m}$ depending on particular type of cable, see Fig. 1. If the transmission bandwidth is narrow the attenuation can be compensated by an additional amplification. A wideband transmission might need even more sophisticated equalization.

\section{B. Galvanic Isolation}

In case of different potentials of sensing ground and the ground of measuring instrument, the galvanic isolation is necessary. Standard galvanic isolation techniques (optocouplers, linear isolation amplifiers, etc.) can hardly satisfy

Manuscript received November 10, 2015, revised January 28, 2016. This research has been supported by the European Regional Development Fund and the Ministry of Education, Youth and Sports of the Czech Republic under the Regional Innovation Centre for Electrical Engineering (RICE), project CZ.1.05/2.1.00/03.0094.

J. Svarny is with the Department of Technologies and Measurements, Faculty of Electrical Engineering / RICE, University of West Bohemia, Pilsen, Czech Republic (phone: +420-377-634-559; fax: +420-377-634-502; e-mail svarny@ket.zcu.cz).

doi: $10.11601 /$ ijates.v5i1.131 the demands for the state of the art analog transmission system. If the barrier in the order of $10 \mathrm{kV}$ is required, the above mentioned techniques are inapplicable and the transmission line has to be based on an optical link.

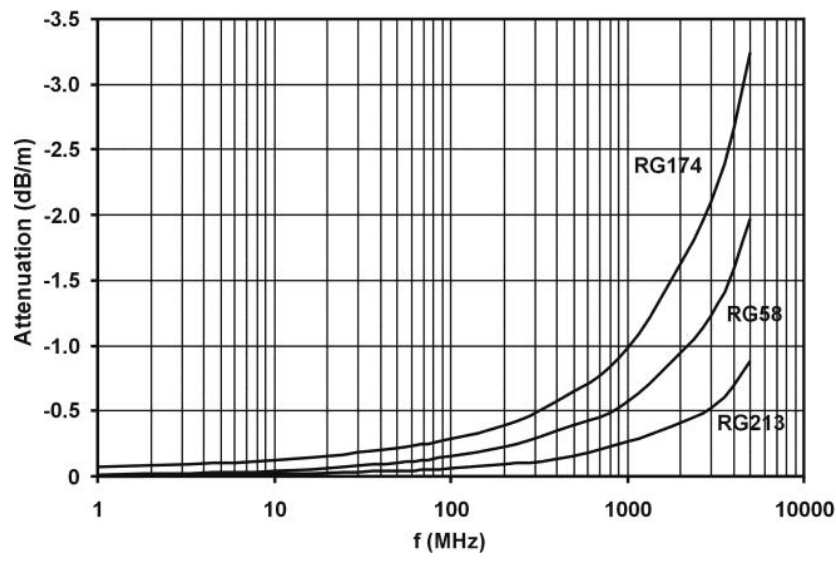

Fig. 1. Typical losses of common coaxial cables.

\section{Measuring Input Protection}

There can be expected some high-frequency (HF) and broadband (BB) measuring instrument at the end of the transmission link. Naturally, most of the HF oscilloscopes and spectrum analyzers use $50 \Omega$ input with very low input voltage immunity (typically of $5 \mathrm{~V}_{\mathrm{pp}}$ ). The transmission system should be designed to provide the proper impedance match and the measuring instrument protection as well.

The paper brings solution of the transmission system that breaks some limits of coaxial cable and meets following requirements: The system has to be able to transmit very weak analog signals in range from $\mu \mathrm{V}$ up to several tens of $\mathrm{mV}$. Primarily, the system is aimed to measure a broadband signal from a sensor installed at the high voltage (HV) test stand and bring it to the broadband analyzer. The analyzer is located outside the HV area (10-100 m away). The input and output must be resistively matched to $50 \Omega$. The overall gain of the transmission system has to be positive $(0 \mathrm{~dB}$ min.). A minimum frequency range of $10 \mathrm{kHz}-2.5 \mathrm{GHz}$ is required. The system must ensure galvanic isolation in the order of $10 \mathrm{kV}$ or more.

Apparently, the only possibility is to use an analog fiber optic link (AFOL). Theory of the AFOL systems based either on directly or externally modulated laser have been discussed in many papers and books, see [2]-[10] for 
instance. The range of applications include: radio signal over fiber distribution, radar connection, remote antenna, EMC testing, signal waveform measurement and monitoring, wideband waveform detection, safe high voltage measurement etc. Some commercially available AFOL systems can be already found on the market (PPM Ltd., Pharad LLC, Teseo S.p.A., Spinner GmbH and other companies). They cover various frequency ranges depending on particular application. Unfortunately, there was not found any system on the market covering the all desired bandwidth (including both lower and upper cut-off frequency simultaneously). This fact was the motivation for development of the own specifically tailored solution.

\section{TheORETICAL REFLECTION}

To achieve adequate bandwidth and gain it was chosen a solution based on an external analog electro-optic modulator of Mach-Zehnder type (MZM), a powerful laser source and a fast photodetector with no post-amplifier.

The modulator works on principle of integrated MachZehnder interferometer [11]. Maximum linearity and low distortion of the signal is got by proper biasing of the MZM at the quadrature operating point [12]. Input optical port is excited by a constant optical power generated by a distributed feedback (DFB) laser diode (LD). The measured signal is amplified and led to the radiofrequency (RF) port of the modulator. The output optical port generates the intensity modulated optical power. Finally, the optical signal is demodulated by a PIN photodiode (PD).

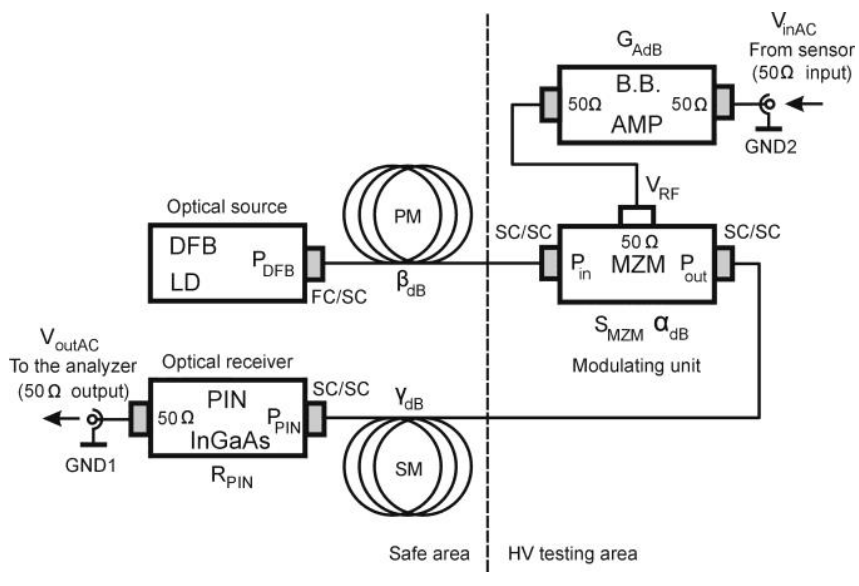

Fig. 2. Block diagram of the transmission system.

A positive overall gain $G_{\mathrm{SdB}}$ (1) of the transmission system is one of the vital features to accomplish with the design.

$$
G_{S d B}=20 \log \frac{V_{\text {outAC }}}{V_{\text {inAC }}} \geq 0 d B
$$

The PIN detector of the receiver is reversely biased and resistively matched to the $\mathrm{R}_{\mathrm{L}}$ load. The $\mathrm{AC}$ component of the receiver output voltage $\mathrm{V}_{\text {outAC }}$ can be expressed by an AC component of a PIN photocurrent $\mathrm{I}_{\text {PINAC }}(2)$.

$$
V_{\text {outAC }}=\frac{I_{P I N A C}}{2} R_{L}
$$

The PIN diode photocurrent $\mathrm{I}_{\mathrm{PINAC}}$ is directly proportional to the detector input optical power. The $R_{P I N}$ (responsivity of the PIN diode) represents the conversion constant between the incident optical power and the photocurrent. If the insertion loss of the single mode (SM) optical cable is $\gamma_{\mathrm{dB}}$ and the $\mathrm{P}_{\text {outAC }}$ stands for the $\mathrm{AC}$ component of the modulator output optical power, the $\mathrm{V}_{\text {outAC }}$ is (3).

$$
V_{\text {out } A C}=\frac{R_{P I N}}{2} R_{L} P_{\text {out } A C} 10^{\frac{\gamma_{d B}}{10}}
$$

Regarding the moderate modulation depth i.e. relatively low input voltage $\mathrm{V}_{\mathrm{RF}}$, the gain of the modulator can be expressed by a constant value $\mathrm{S}_{\mathrm{MZM}}$. $\mathrm{V}_{\text {outAC }}$ is (4) then.

$$
V_{\text {outAC }}=\frac{R_{P I N}}{2} R_{L} 10^{\frac{\gamma_{d B}}{10}} S_{M Z M} V_{R F}
$$

The $S_{\text {MZM }}$ parameter represents the slope efficiency (5) of the modulator for the positive quadrature operating point [13]. From mathematical point of view it is a tangent at the inflexion point of the modulator transfer chart, see Fig. 3.

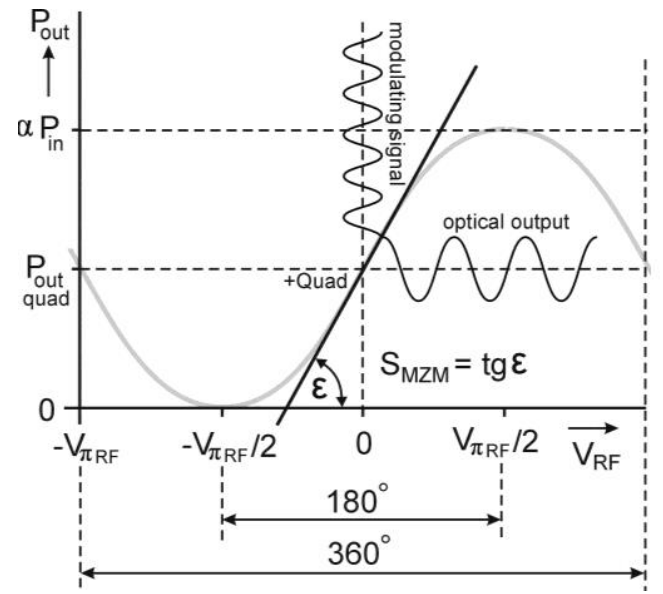

Fig. 3. Slope efficiency of the MZM biased to the positive quadrature point

$$
S_{M Z M}=\frac{d P_{o u t}}{d V_{R F}}=\operatorname{tg} \varepsilon=\frac{P_{i n} 10^{\frac{\alpha_{d B}}{10}} \pi}{2 V_{\pi R F}}
$$

The $\mathrm{P}_{\text {in }}$ is the input optical power, the $\alpha_{\mathrm{dB}}$ is the insertion loss of fully open modulator and the $\mathrm{V}_{\pi \mathrm{RF}}$ is half-wave voltage of the RF port of the MZM. Provided there is used a pre-amplifier with the $\mathrm{G}_{\mathrm{AdB}}$ gain in front of the MZM RF input, the equation (4) will expand to (6).

$$
V_{\text {outAC }}=\frac{R_{P I N}}{2} R_{L} 10^{\frac{\gamma_{d B}}{10}} \frac{P_{i n} 10^{\frac{\alpha_{d B}}{10}} \pi}{2 V_{\pi R F}} 10^{\frac{G_{A d B}}{20}} V_{i n A C}
$$


Finally, taking into account the insertion loss of the input polarization maintenance $(\mathrm{PM})$ optical cable $\beta_{\mathrm{dB}}$, the overall gain of the electro-optic transmission system $\mathrm{G}_{\mathrm{SdB}}$ can be expressed as (7).

$$
\begin{gathered}
G_{S d B}=G_{A d B}+2\left(\alpha_{d B}+\beta_{d B}+\gamma_{d B}\right)+ \\
+20 \log \frac{R_{P I N} R_{L} P_{D F B} \pi}{4 V_{\pi R F}}
\end{gathered}
$$

Parameters $R_{P I N}, R_{L}, \alpha_{d B}, \beta_{d B}, \gamma_{d B}, V_{\pi R F}$ are almost invariant constants regardless the choice of particular components. Nevertheless, the system gain $\mathrm{G}_{\mathrm{SdB}}$ can be affected by the pre-amplifier gain $\mathrm{G}_{\mathrm{AdB}}$ and by the optical power $\mathrm{P}_{\mathrm{DFB}}$ as well. Fig. 4 depicts dependency of minimum needed gain of the RF pre-amplifier as a function of the laser optical power $\mathrm{P}_{\mathrm{DFB}}$ in order to obtain the lossless transmission $\left(\mathrm{G}_{\mathrm{SdB}}=0 \mathrm{~dB}\right)$. The dependency was calculated for typical values of system components: $\mathrm{R}_{\mathrm{PIN}}=0.7 \mathrm{~A} / \mathrm{W}$ (InGaAs photodetector @ $1550 \mathrm{~nm}$ ), $\mathrm{R}_{\mathrm{L}}=50 \Omega, \beta_{\mathrm{dB}}=$ $-0.5 \mathrm{~dB}$ (PM patch-cord), $\gamma_{\mathrm{dB}}=-0.3 \mathrm{~dB}$ (SM patch-cord), $\mathrm{V}_{\pi \mathrm{RF}}=3.7 \mathrm{~V}, \alpha_{\mathrm{dB}}=-3 \mathrm{~dB}$ (FA20 modulator).

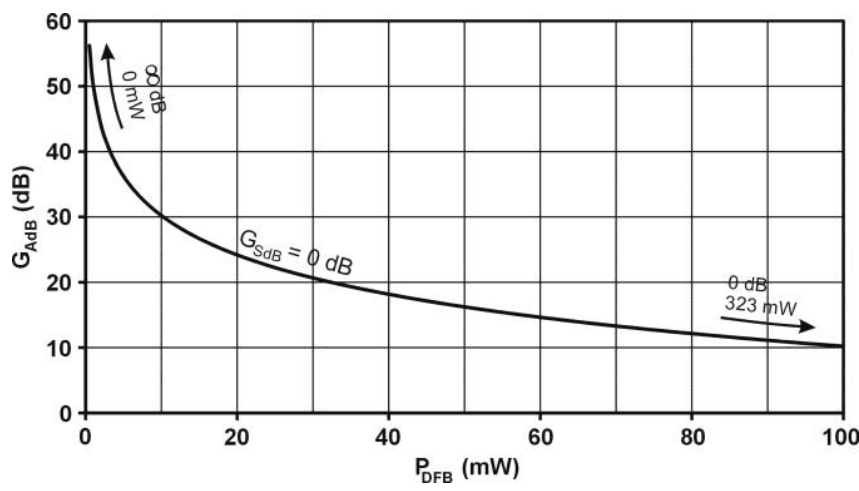

Fig. 4. Needed pre-amplification $\mathrm{G}_{\mathrm{AdB}}$ as a function of $\mathrm{P}_{\mathrm{DFB}}$ for lossless transmission $\left(\mathrm{G}_{\mathrm{SdB}}=0 \mathrm{~dB}\right)$.

Advantageously, the gain of the RF pre-amplifier can be decreased at the expense of the laser power. Unfortunately, this rule has some limits in fact. Probably the most severe one is inherent in maximum acceptable input optical power of the PIN detector $\mathrm{P}_{\text {PINmax }}$. That is why, it is desirable to search for a detector that covers specified frequency range and is able to handle highest possible input power at the same time. The detector that was chosen has $\mathrm{P}_{\mathrm{PINmax}}=$ $10 \mathrm{~mW}$. As the $\mathrm{P}_{\text {PINmax }}$ value is defined, the maximum permissible laser power $\mathrm{P}_{\mathrm{DFBmax}}$ can be stipulated by (8).

$$
P_{D F B \max }=P_{P I N \max } 10^{-\left(\frac{\alpha_{d B}+\beta_{d B}+\gamma_{d B}}{10}\right)}
$$

The maximum acceptable optical power $\mathrm{P}_{\mathrm{DFBmax}}$ for safe operation of the PIN photodiode works out $24 \mathrm{~mW}$. Consequently, in accordance with the Fig. 4, there is necessity to ensure $22.6 \mathrm{~dB}$ pre-amplification at minimum.

\section{THE SYSTEM IMPLEMENTATION}

\section{A. Stabilized Optical Source}

The pigtailed $20 \mathrm{~mW} / 1550 \mathrm{~nm}$ DFB laser module A1905LMI [14] was used as a source of optical radiation. In addition to the LD itself, the laser module is equipped with a thermoelectric cooler, a NTC thermistor, a monitor $\mathrm{PD}$ and an optical isolator. The module is pigtailed with the PM fiber ended with standard FC/PC connector.

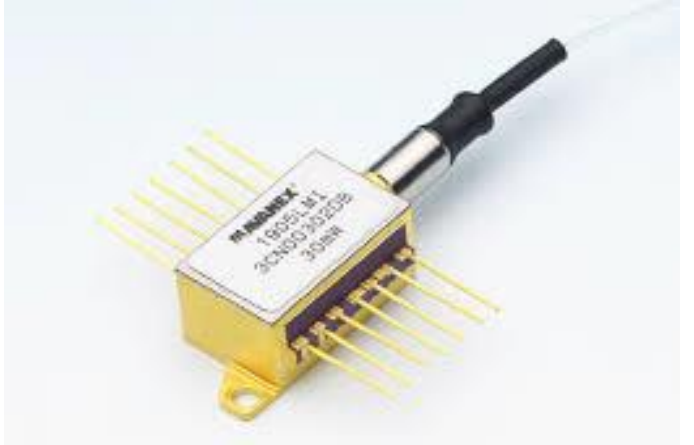

Fig. 2. DFB laser module A1905LMI.

In order to achieve desired behavior of the LD, there was necessary to design and implement effective LD drivers. The LD is operated in automatic current control regime (ACC). Simultaneously, the LD temperature is controlled by a thermoelectric cooler controller (TECC). The ACC controller is based on WLD3343 hybrid IC (Wavelength Electronics, Inc.). The TECC uses LTC1923 IC (Linear Technology, Inc.). Both the drivers are controlled by the Control Unit equipped with AT89S53 (Atmel, Inc.) microcontroller. The control unit coordinates operation of the controllers and monitors vital parameters of the laser module.

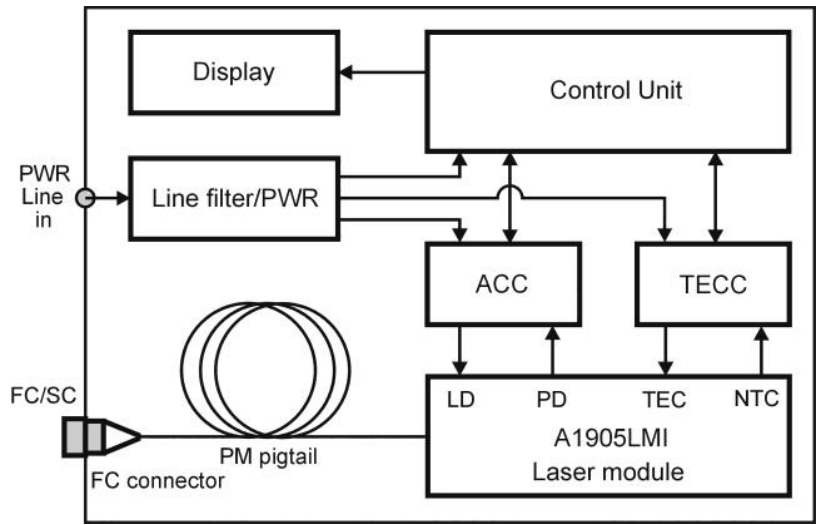

Fig. 6. Block diagram of the stabilized optical source.

A digital optical multimeter OMM6810B (by ILX Lightwave, Inc.) with InGaAs sensing head OMH6727B was used to verify the output optical power value and the stability of the laser source. It was found that the optical source generates power of $21.68 \mathrm{~mW}$ (i.e. tightly below acceptable maximum). 


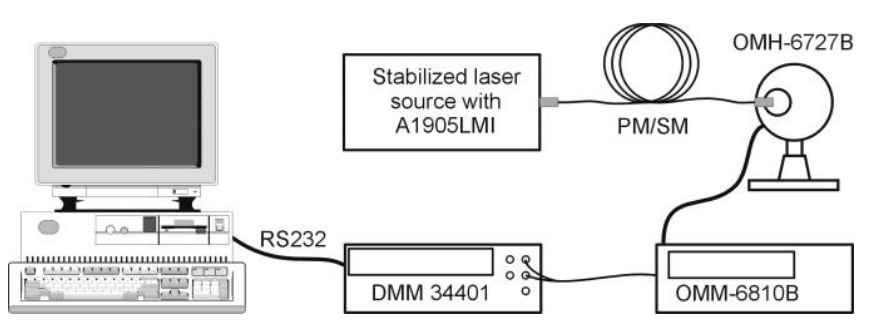

Fig. 7. The verification of the stabilized optical source.

The power fluctuation measurement was done using the Power Analog Out which is accessible on the rear panel of the OMM6810B. The Power Analog Out provides the amplified photocurrent generated by OMH6727B. An Agilent 34401 DMM was used to digitize the value. The data were measured with sampling rate of $2 \mathrm{Sa} / \mathrm{s}$. The measurements revealed fluctuation $\Delta \mathrm{P}_{\text {out }}= \pm 12.5 \mu \mathrm{W}$ $( \pm 0.003 \mathrm{~dB})$ oscillating around the average value of $21.68 \mathrm{~mW}$ (see Fig. 8.).

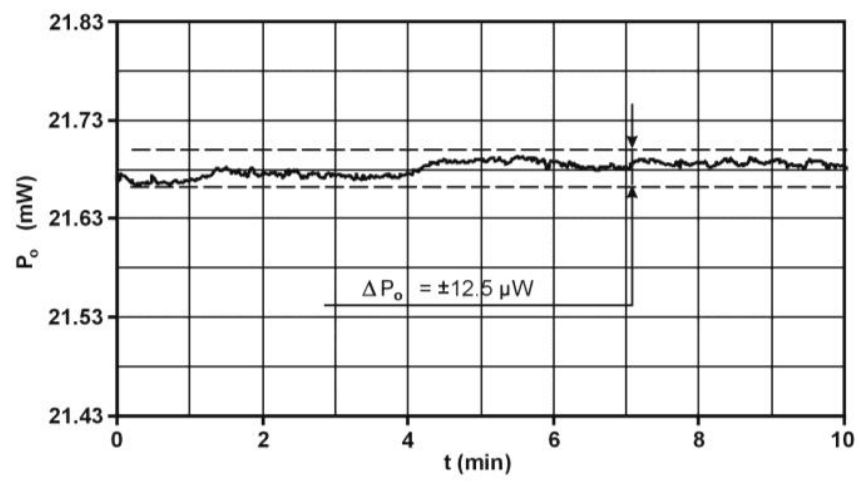

Fig. 8. Short-term stability measurements of the optical source.

Similar measurements were done to evaluate long-term stability. Result of the measurements can be seen in Fig. 9. The 3-hour-period of measurements revealed fluctuation $\Delta \mathrm{P}_{\text {out }}= \pm 45 \mu \mathrm{W}( \pm 0.009 \mathrm{~dB})$.

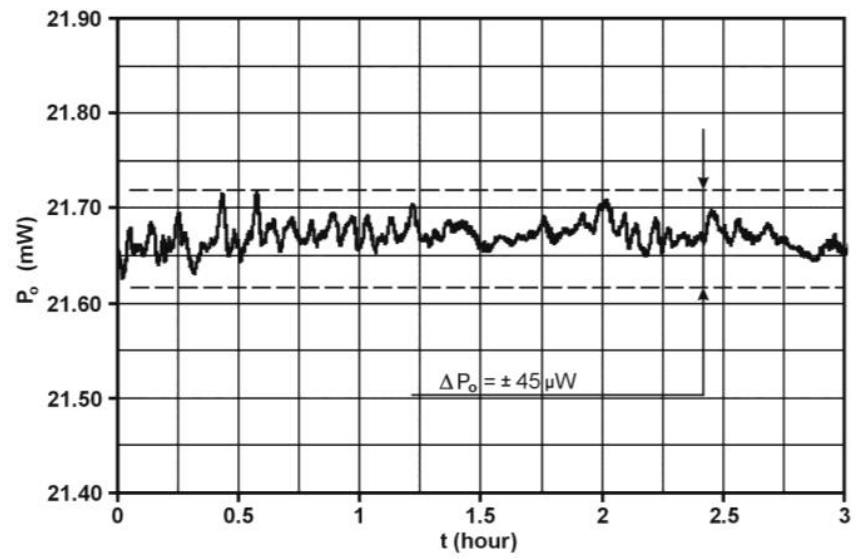

Fig. 9. Long-term stability measurements of the optical source.

The detailed description of the laser unit including the laser evaluation can be found in [15].

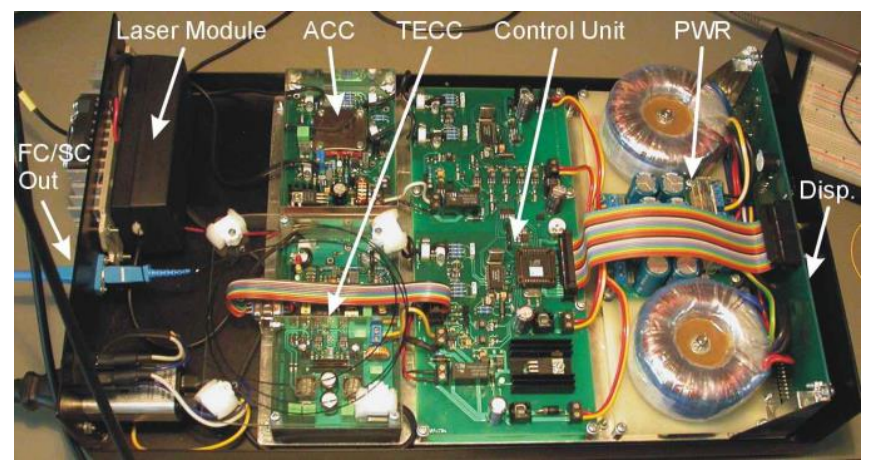

Fig. 10. Stabilized optical source.

\section{B. External Modulating Unit}

Analog intensity electro-optic modulator FA20 [16] forms a key part of the modulating unit. It is the interferometric modulator integrated in the $\mathrm{LiNbO}_{3}$ substrate. The modulator has a standalone bias port for the operating point control and the modulating input (RF port). Besides that, the modulator is equipped with an integrated PD to monitor the output optical power. Bandwidth of the device is 13.7 $\mathrm{GHz}(-3 \mathrm{~dB})$. Half-wave voltages are agreeably low (3.7V @ RF port and 5.2 V @ bias port). Extinction ratio is relatively high $(31.4 \mathrm{~dB})$.

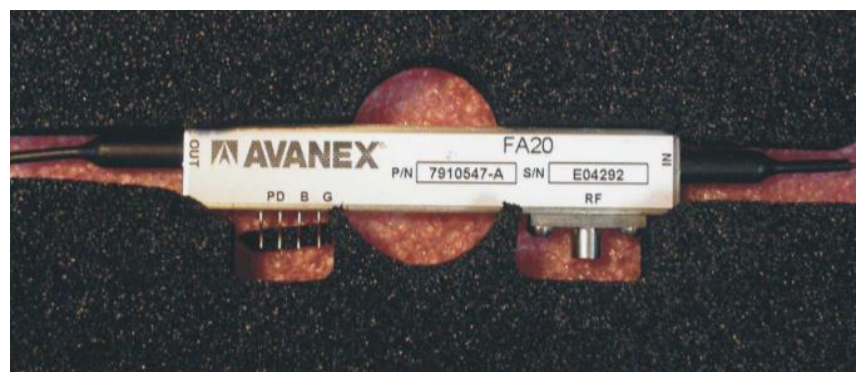

Fig. 11. The FA20 analog intensity modulator.

Inner circuitry of the modulating unit consists of three parts (a MZM baseboard, a RF pre-amplifier \& power supply block and a bias driver block). The MZM baseboard comprises the modulator, protection circuits, a bias port buffer and a transimpedance amplifier of the MZM inner PD. The power supply block (battery pack) is integrated with a charger and the low-noise, broadband pre-amplifier ABL0300 (9 kHz-3 GHz) [17]. Nominal gain of the preamplifier $(32 \mathrm{~dB})$ has a sufficient headroom over the calculated $\mathrm{G}_{\mathrm{AdB}}$ minimum. 


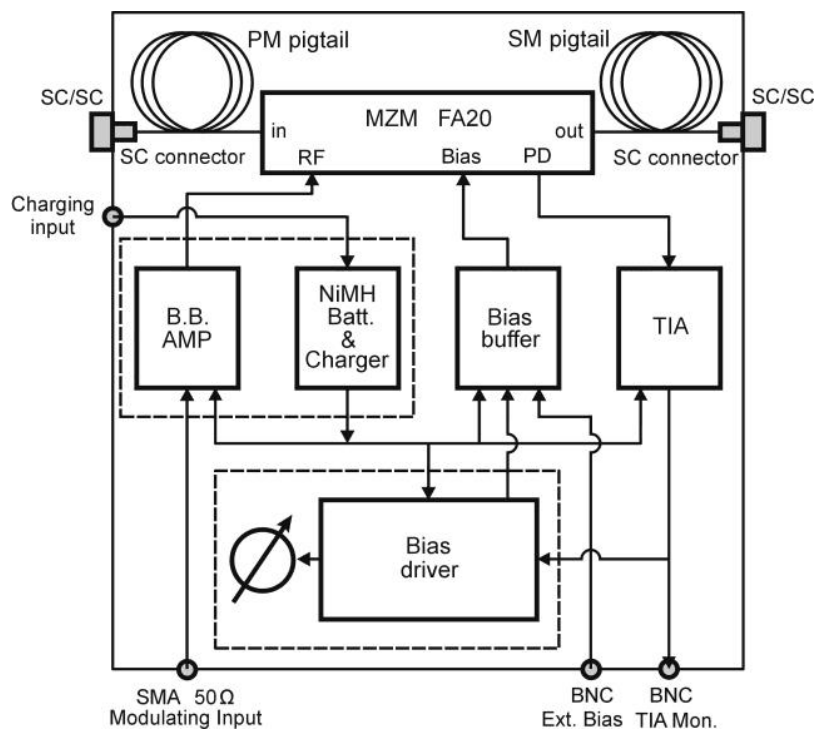

Fig. 12. Block diagram of the external modulating unit.

Desired response of the MZM is achieved by proper adjustment of the operating point to the quadrature position. This task is solved by the bias driver. The bias driver takes advantage of known frequency response of the modulator biased at the proximity of quadrature point. Provided the input bias voltage is formed by DC component $\mathrm{V}_{\mathrm{DC}}$ and superposed $\mathrm{AC}$ harmonic component with $\mathrm{V}_{\mathrm{AC}}$ amplitude (9), the output optical power $\mathrm{P}_{\mathrm{o}}$ of the MZM can be described by (10).

$$
\begin{gathered}
V_{i n}=V_{D C}+V_{A C} \sin \left(\omega_{A C} t\right) \\
P_{o}=\frac{\alpha P_{i n}}{2}+\frac{\alpha P_{i n}}{2} \cos \left(\frac{V_{D C}}{V_{\pi}} \pi\right) J_{0}\left(\frac{V_{A C}}{V_{\pi}} \pi\right)+ \\
+\alpha P_{i n} \cos \left(\frac{V_{D C}}{V_{\pi}} \pi\right) \sum_{k=1}^{\infty} J_{2 k}\left(\frac{V_{A C}}{V_{\pi}} \pi\right) \cos \left(2 k \omega_{A C} t\right)- \\
-\alpha P_{i n} \sin \left(\frac{V_{D C}}{V_{\pi}} \pi\right) \sum_{k=1}^{\infty} J_{2 k-1}\left(\frac{V_{A C}}{V_{\pi}} \pi\right) \sin \left[(2 k-1) \omega_{A C} t\right],
\end{gathered}
$$

where $P_{\text {in }}$ is the input optical power of the MZM, $\alpha$ is the insertion loss of the MZM, $\mathrm{V}_{\pi}$ is half-wave voltage of the bias port of the MZM and $\mathrm{J}_{\mathrm{k}}(\mathrm{x})$ is a $\mathrm{k}^{\text {th }}$ order Bessel function. For detailed derivation see [18]. From the equation (10) formulas for computing the magnitude level of particular $\mathrm{n}^{\text {th }}$ harmonic component (11), (12) can be easily extracted.

$$
P_{o-n-o d d}=\left|\alpha P_{i n} \sin \left(\frac{V_{D C}}{V_{\pi}} \pi\right) J_{n}\left(\frac{V_{A C}}{V_{\pi}} \pi\right)\right|,
$$

where $n \in\langle 1,3,5, \ldots\rangle$

$$
P_{o-n-\text { even }}=\left|\alpha P_{i n} \cos \left(\frac{V_{D C}}{V_{\pi}} \pi\right) J_{n}\left(\frac{V_{A C}}{V_{\pi}} \pi\right)\right|,
$$

where $n \in\langle 2,4,6, \ldots\rangle$
Equations (11) and (12) can be used to visualize the levels of particular harmonic components at the output of MZM as a function of DC level at the bias port. The amplitudes of the first three harmonics were depicted in Fig. 13. The curves were computed for input optical power $P_{\text {in }}=$ $21.68 \mathrm{~mW}$, the FA20 parameters $\left(\alpha=0.5, \mathrm{~V}_{\pi}=5.2 \mathrm{~V}\right)$ and modulating voltage $\mathrm{V}_{\mathrm{AC}}=500 \mathrm{mV}$.

It is clear that the magnitude of $2^{\text {nd }}$ harmonic component reaches its minimum just at the quadrature point. Moreover, the $2^{\text {nd }}$ harmonic reflects the position of the desired operating point around the quadrature region with an extraordinary sensitivity. It brings the possibility to use the $2^{\text {nd }}$ harmonic component as an indicator of proper setup. The implemented bias driver utilizes the phenomenon.

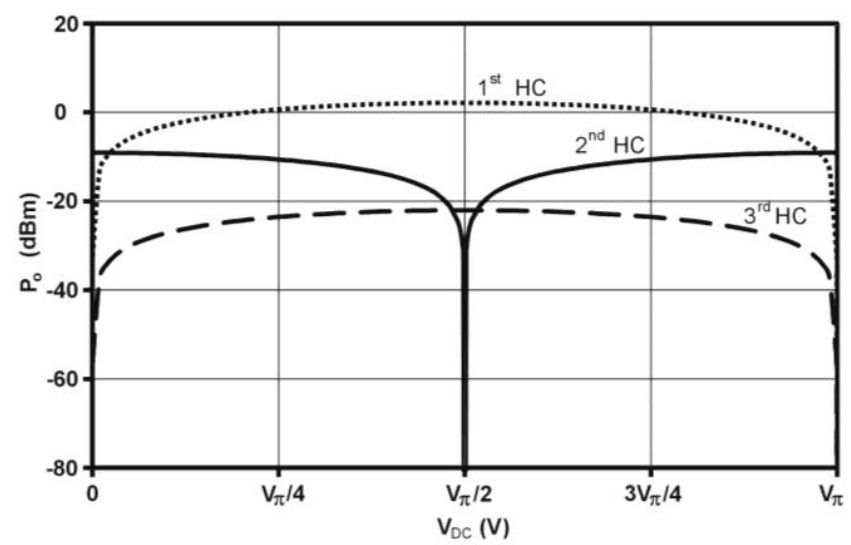

Fig. 13. Dependency of $1^{\text {st }}, 2^{\text {nd }}$ and $3^{\text {rd }}$ harmonics on DC bias $V_{D C}$.

The bias input port of the modulator is excited by a manually adjustable DC level and superimposed LF harmonic signal. Simultaneously, the inner PD of the MZM detects output signal of the modulator. Following circuitry analyses presence of $2^{\text {nd }}$ harmonic component in the spectrum of detected signal. The detailed structure of the circuit is depicted in Fig. 14.

The DC level is generated by a voltage reference (VREF) and manually adjustable divider (DC Bias). The AC part generates $1 \mathrm{kHz}$ sinus signal with amplitude of $500 \mathrm{mV}$. Purity of the AC signal is important. That is why there was used method based on filtration of square wave signal. The quartz oscillator (XO) generates $4.096 \mathrm{MHz}$ digital signal that is divided and shaped by a binary 12-stage ripple counter (DIV). As the spectrum of the generated square wave consists of odd harmonics only, the problem of absence of second harmonic is resolved spontaneously then. The higher odd harmonics are cut-off by a narrow bandpass filter BPF1 with center frequency of $1 \mathrm{kHz}$.

The signal form BPF1 block is mixed with DC level from the DC Bias block by means of summing amplifier $\Sigma_{1}$. The following stage gives a $\pi$ phase shift to the signal. This is vital for analysis of the signal detected by the inner monitor photodiode PD.

The measuring chain has to analyze presence of $2^{\text {nd }}$ harmonic in the output spectrum and visualize its magnitude. The inner PD of the MZM generates current that is inversely proportional to the output optical power of the 


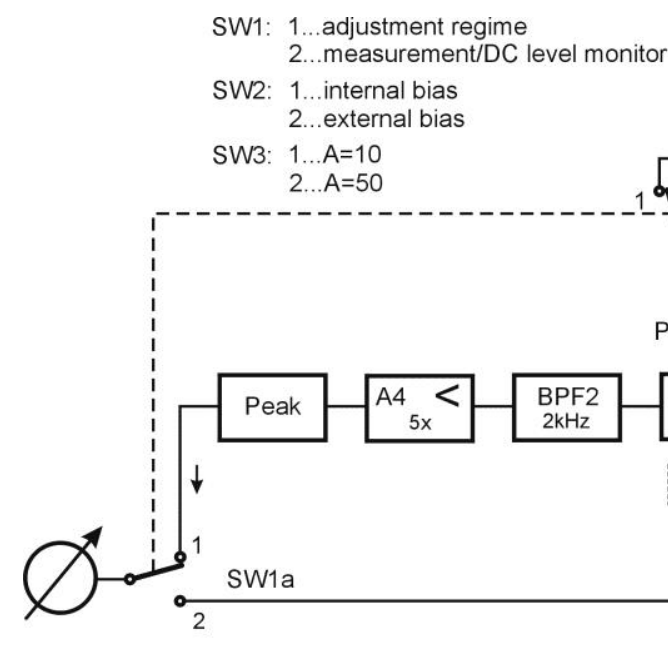

Fig. 14. Block diagram of the bias driver.

The signal of the PD is conditioned by means of transimpedance amplifier (TIA). Gain of the TIA was experimentally set to $-470 \mathrm{~V} / \mathrm{mA}$. The DC level of the TIA output signal is excluded by amplifier with integrating feedback (AC couple stage). To derive $2^{\text {nd }}$ harmonic from the spectrum, there is necessary to eliminate fundamental in received spectrum at first. This is carried out by summing amplifier $\Sigma_{2}$. Due to the phase shift of the received signal the summing amplifier $\Sigma_{2}$ works as a difference amplifier in fact. At the output of the $\Sigma_{2}$ the difference between the received $\mathrm{AC}$ signal and the exciting signal can be found. (As there has to be set-up certain level of attenuation of exciting signal to achieve substantial decimation of fundamental at the output of $\Sigma_{2}$ block the exciting signal is attenuated by the A2 block.) The signal is amplified by the A3 amplifier and filtered by BPF2 filter then. The BPF2 stage forms a band-pass filter tuned to $2^{\text {nd }}$ harmonic of the exciting signal. After an additional amplification in A4 block the signal is detected by a peak detector (Peak) and sent to the deflective measuring instrument.

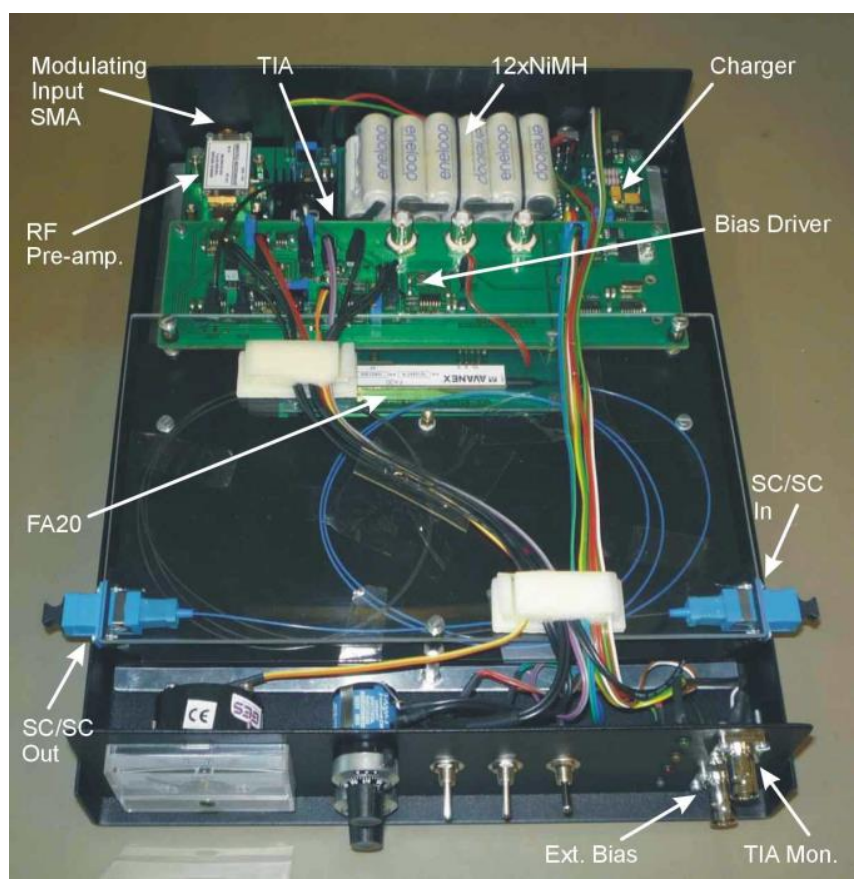

Fig. 15. External modulating unit.

\section{A. Receiver}

The receiver is formed by an optical detector which works as a demodulator. The detector converts the intensity modulated optical power to an electrical signal. Wide bandwidth and high linearity of the receiver is achieved thanks to operation in passive regime (i.e. with no postamplifier).

The overall gain of the transmission system is concentrated partially in the RF pre-amplifier of the modulator and partially in the high optical power of the laser. That is why it was necessary to use a detector with the linear response up to $10 \mathrm{~mW}$ of input optical power. There was used a special broadband telecommunication PIN photodiode 2522A (Emcore, Inc.) [19]. The PD is working with a negative bias and is internally resistively matched to $50 \Omega$. Bandwidth of the photodiode is DC$15 \mathrm{GHz}$ and minimum responsivity is $0.7 \mathrm{~A} / \mathrm{W} @ 1550 \mathrm{~nm}$. The photodiode module is equipped with the SM pigtail ended with standard SC/PC connector.

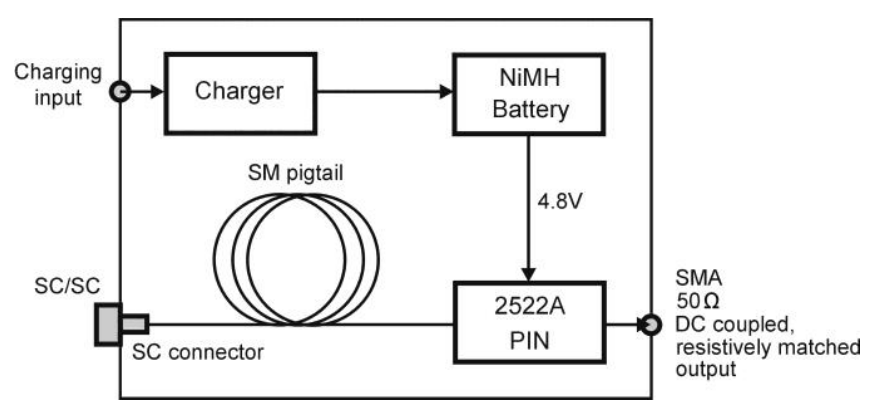

Fig. 16. Block diagram of the receiver.

The receiver uses a battery pack of $4 \mathrm{NiMH}$ cells as a power supply. A battery charger is integrated on the circuit board. Low supply voltage and galvanic isolation from the rest of the system, make the receiver the absolutely safe source of signal to a sensitive frontend of any prospective $\mathrm{BB}$ analyzer or a HF oscilloscope. 


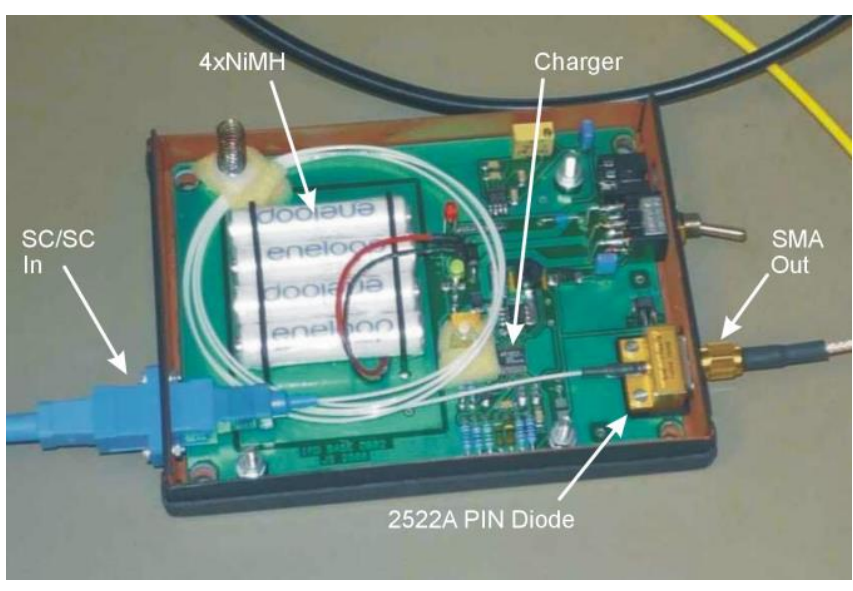

Fig. 17. Broadband optical receiver.

\section{Results}

Based on theoretical consideration there were designed and implemented all the three parts of the broadband electrooptic transmission system. Subsequently, measurements of the frequency response were carried out. Lower cut-off frequency was tested by the oscilloscope. Upper part of the range (above $300 \mathrm{kHz}$ ) was tested by the ZVB8 network analyzer.

The measured frequency range of the system is $9 \mathrm{kHz}-$ $3.45 \mathrm{GHz}(-3 \mathrm{~dB})$. In accordance with (7), the theoretical value of the overall gain should be $8.5 \mathrm{~dB}$. Nevertheless, the measurement revealed the average gain of $6 \mathrm{~dB}$. Frequency chart is relatively flat and well balanced $(6 \mathrm{~dB} \pm 1.5 \mathrm{~dB})$.

The difference between expected and real value of the gain is relatively small $(2.5 \mathrm{~dB})$. The divergence can be justified by a sum of several factors. Firstly, the insertion loss of the optical patch-cords may slightly differ from the nominal value. Furthermore, to ensure demountable connection between the units there have been implemented the SC/SC optical cable adapters. Two of them can be found between the laser and the modulator and the next two between the modulator and the detector. Each of the adapters has power to bring an additional insertion loss uncertainty. Last but not least, it must be kept in the mind that all the calculations were made on basis of typical data sheet parameters that may slightly differ from reality.

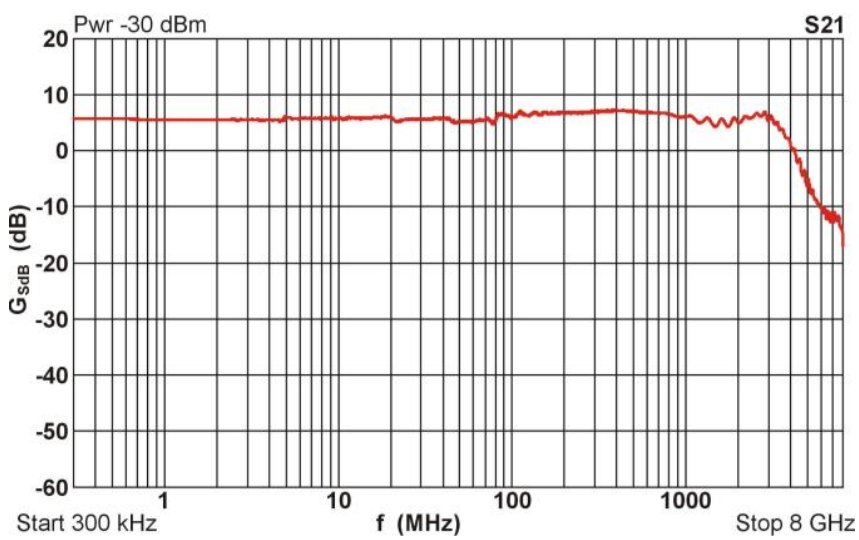

Fig. 18. The frequency response of the transmission system measured by the ZVB8 network analyzer.

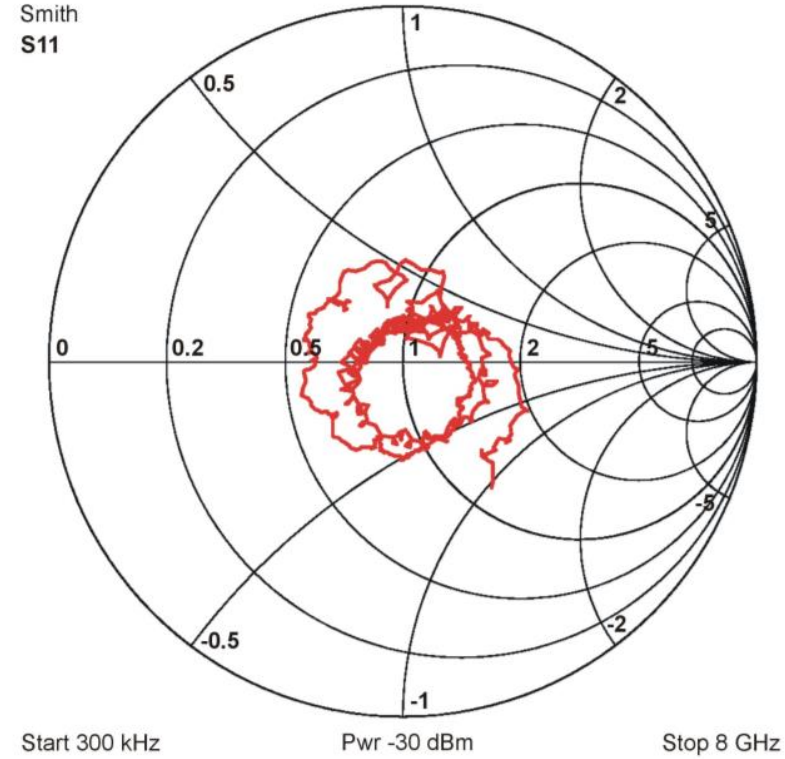

Fig. 19. The Smith chart of S11 parameter of the transmission system measured by the ZVB8 network analyzer.

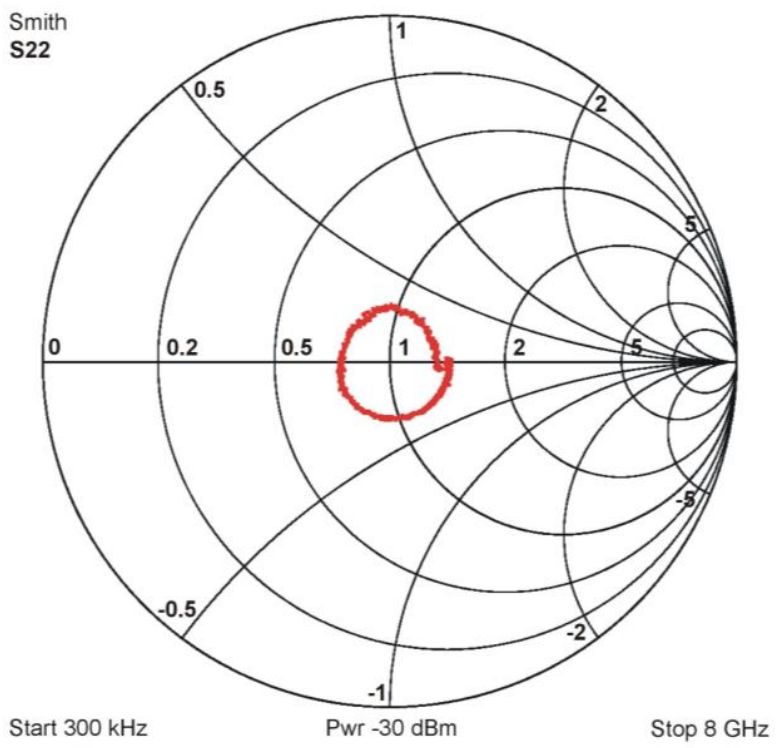

Fig. 20. The Smith chart of S22 parameter of the transmission system measured by the ZVB8 network analyzer.

The network analyzer was also used to evaluate the input and output impedance matching of the designed transmission system. There were measured S11 and S22 parameters (Fig. 19, Fig. 20). The values of the measured $\mathrm{S}$-parameters were used to calculate the voltage standing wave ratio (VSWR) for input and output port respectively and for particular termination of the system, see Fig. 21 and Fig. 22. 


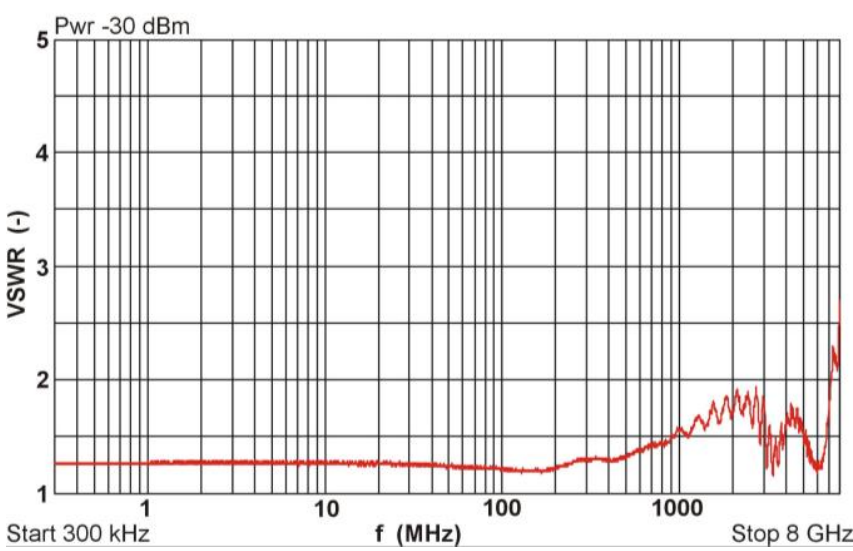

Fig. 21. The voltage standing wave ratio for the transmission system input port ( $50 \Omega$ termination of the input).

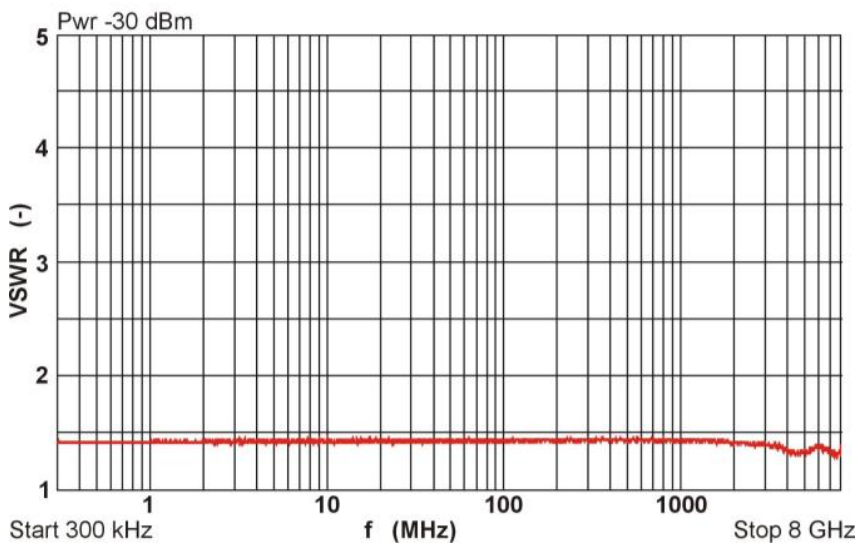

Fig. 22. The voltage standing wave ratio for the transmission system output port ( $50 \Omega$ termination of the output).

Taking into account the upper cut-off frequency of the system $(3.45 \mathrm{GHz})$, the matching of the input to the $50 \Omega$ source is characterized with VSWR of $1.2: 1$ up to $1.9: 1$. The matching of the output of the system to the $50 \Omega$ load is characterized with VSWR of 1.3:1 up to $1.4: 1$.

TABLE I

COMPARISON OF THE RESULTS

\begin{tabular}{llcccc}
\hline \hline \multicolumn{1}{c}{ System } & Company & $\begin{array}{c}\text { Bandwidth } \\
{[\mathrm{MHz}]}\end{array}$ & $\begin{array}{c}\text { Gain } \\
{[\mathrm{dB}]}\end{array}$ & $\begin{array}{c}\text { Flatness } \\
{[\mathrm{dB}]}\end{array}$ & $\begin{array}{c}\text { VSWR } \\
{[-]}\end{array}$ \\
\hline K series [20] & PPM & $0.002-1350$ & 0 & \pm 3.0 & 1.5 \\
S series [20] & PPM & $10-3000$ & 0 & \pm 1.5 & 1.5 \\
$\begin{array}{l}\text { Eco Line [21] } \\
\text { PXR001-190 }\end{array}$ & Spinner & $50-2400$ & 5 & \pm 0.5 & 2 \\
[22] & Pharad & $3-19000$ & 15 & \pm 2.0 & 2 \\
$\begin{array}{l}\text { PXR005-030 } \\
{[23]}\end{array}$ & Pharad & $500-3000$ & 0 & \pm 1.0 & 2 \\
$\begin{array}{l}\text { OAM208 [24] } \\
\begin{array}{l}\text { Presented } \\
\text { solution }\end{array}\end{array}$ & Teseo & $0.5-3000$ & 0 & \pm 4.0 & 1.4 \\
\hline \hline
\end{tabular}

Table I resumes a brief comparison of our developed system (last line) with the similar systems that are commercially available on the market. Apparently, the main advantage of our system is inherent in the remarkably favorable lower cut-off frequency and relatively high gain. The VSWR column gives the worst case values across the frequency range of the particular system. In case of our solution the VSWR value was mostly affected by behavior of the input port at the very end of the frequency range. For bottom and middle part of bandwidth of the system (up to
$1 \mathrm{GHz}$ approximately) the impedance matching is apparently better. Across the measured bandwidth the VSWR of the output port itself works out significantly better in comparison to the presented value as well.

\section{CONCLUSION}

There was designed and implemented unique analog transmission system for the safe connection of the HF $2.5 \mathrm{GHz}$ oscilloscope to a sensor which works at very high potential. Problem of ultimate galvanic isolation and oscilloscope protection was solved by implementation of an optical fiber as the transmission media and battery-powered units. Unlike the metallic cable the length of the used medium can be prolonged up to hundreds of meters with virtually no impact to the signal quality. Upper and lower cut-off frequencies are mostly given by the broadband RF pre-amplifier features. (The frequency range of the modulator and the detector itself is much higher.) Thanks to the external modulation, the overall gain of the system would be increased by an additional increase of the laser power. Unfortunately, the method assumes use of a detector with even higher input power capability. Because such a detector is nowadays unavailable, the system works at its optical power maximum.

\section{REFERENCES}

[1] Coaxial Cable Attenuation ratings, National Wire and Cable Corp., [Online]. Available: http://www.nationalwire.com/pdf/COAXIAL-ATTENUATION.pdf

[2] E. I. Ackerman, C. H. Cox III, "RF fiber-optic link performance," IEEE Microwave Magazine, vol. 2, pp. 50-58, December 2001.

[3] C. Cox III, E. Ackerman, R. Helkey, G. E. Betts, "Techniques and performance of intensity-modulation direct-detection analog optical link," IEEE Trans. on Microwave Theory and Techniques, vol. 45, no. 8, pp. 1375-1383, August 1997.

[4] E. I. Ackerman, W. K. Burns, G. E. Betts, J. X. Chen, J. L. Prince, M D. Regan, H. V. Roussell, C. H. Cox III, "RF-over-fiber links with very low noise figure," IEEE Journal of Lightwave Technology, vol. 26, no. 15, pp. 2441-2448, August 2008.

[5] C. H. Cox III, E. I. Ackerman, G. E. Betts, J. L. Prince, "Limits on the performance of RF-over-fiber links and their impact on device design," IEEE Transactions on Microwave Theory and Techniques, vol. 54, no. 2, February 2006.

[6] I. L. Newberg, "High-performance lossless RF fiber optic link," in Proc. International Conference on Phased Array Systems and Technology, Dana Point, CA, 2000, pp. 375-378.

[7] E. I. Ackerman, C. H. Cox III, "State of the art in analog fiber-optic link technology," in Proc. URSI International Symposium on Signals, Systems and Electronics ISSSE98, Pisa, 1998, pp. 372-377.

[8] J. M. Senior, Optical Fiber Communications-Principles and Practice, Prentice Hall, 1992.

[9] C. H. Cox III, Analog Optical Links-Theory and Practice, Cambridge University Press, 2004.

[10] A. Brillant, Digital and Analog Fiber Optic Communications for CATV and FTTX Applications, SPIE Press, 2008.

[11] A. Chen, E. J. Murphy, Broadband optical Modulators-Science, Technology and Applications, CRC Press, 2012.

[12] A. Hilt, "Microwave harmonic generation in fiber-optical links," Journal of Telecommunications and Information Technology, vol.1/2002, pp. 22-28, 2002.

[13] J. Svarny, "Analysis of quadrature bias-point drift of Mach-Zehnder electro-optic modulator," in Proc. International 12th Biennial Baltic Electronics Conference BEC2010, Tallinn, 2010, pp. 231-234.

[14] PowerSource ${ }^{T M}$ 1905LMI CW laser module with optical isolator, Up to $30 \mathrm{~mW}, \mathrm{C}$-Band, Avanex Inc., 2005, [Online]. Available: http://www.lightwavestore.com/product_datasheet/OSC-LDPM-C011C_pdf1.pdf 
[15] J. Svarny, "Highly stable $20 \mathrm{~mW}$ infrared laser source," in Proc. International Conference Applied Electronics 09, Pilsen, 2009, pp. 249-252.

[16] PowerLog ${ }^{T M}$ FA-20, $20 \mathrm{GHz}$ Analog Intensity Modulator, Avanex Inc., 2009, [Online]. Available: http://www.amstechnologies.com/fileadmin/amsmedia/downloads/3510 _Oclaro_PowerLog_FA20_v2_1.pdf

[17] Broadband Low Noise Amplifier ABL0300-00-3230, Wenteq Microwave Corp., [Online]. Available: http://www.wenteq.com/Amplifier-PDF/ABL0300-00-3230.pdf

[18] J. Svarny, "Specialized Compact External Electro-Optic Modulating Unit," in Proc.36th International Conference on Telecommunications and Signal Processing TSP2013, Rome, 2013, pp. 227-230.

[19] 2522 Microwave Packaged Photodiode, Emcore Corp., 2007, [Online]. Available: http://www.emcore.com/wp-content/uploads/25221.pdf

[20] Point2point-AC Coupled Fiber Optic Link, PPM Ltd., 2011, [Online]. Available: http://ppmtest.com/wp-content/uploads/point2point-AC-Link-DatasheetPAx-DS-7.pdf

[21] Eco Line Analog Fiber Optic Link, Spinner GmbH, 2008, [Online]. Available: http://www.spinner-group.com/upload/de_D_FO_ECO_304.pdf

[22] $\mathrm{HF}$ - K-band Photonic Transceiver $0.003 \mathrm{GHz}-19 \mathrm{GHz}$, Pharad, 2013, [Online]. Available: http://www.pharad.com/pdf/hf-k-band-rfphotonic-transceiver-datasheet.pdf

[23] UHF - S-band Photonic Transceiver 0.5 GHz-3 GHz, Pharad, 2013 , [Online]. Available: http://www.pharad.com/pdf/uhf-s-band-rf-photonictransceiver-datasheet.pdf

[24] OAM208 Optical Acquisition Module $500 \mathrm{kHz}-3 \mathrm{GHz}$, Teseo S.p.A. 2007, [Online]. Available:

http://www.teseo.net/lang1/files/ds294ab.pdf 